\title{
Production of high-quality electron bunches by dephasing and beam loading in channeled and unchanneled laser plasma accelerators
}

\author{
Citation for published version (APA): \\ Geddes, C. G. R., Tóth, C., van Tilborg, J., Esarey, E. H., Schroeder, C. B., Bruhwiler, D., Nieter, C., Cary, J., \& \\ Leemans, W. P. (2005). Production of high-quality electron bunches by dephasing and beam loading in \\ channeled and unchanneled laser plasma accelerators. Physics of Plasmas, 12(5), 056709-1/10. \\ https://doi.org/10.1063/1.1882352
}

DOI:

$10.1063 / 1.1882352$

Document status and date:

Published: 01/01/2005

\section{Document Version:}

Publisher's PDF, also known as Version of Record (includes final page, issue and volume numbers)

\section{Please check the document version of this publication:}

- A submitted manuscript is the version of the article upon submission and before peer-review. There can be important differences between the submitted version and the official published version of record. People interested in the research are advised to contact the author for the final version of the publication, or visit the DOI to the publisher's website.

- The final author version and the galley proof are versions of the publication after peer review.

- The final published version features the final layout of the paper including the volume, issue and page numbers.

Link to publication

\footnotetext{
General rights

- You may freely distribute the URL identifying the publication in the public portal. follow below link for the End User Agreement:

www.tue.nl/taverne

\section{Take down policy}

If you believe that this document breaches copyright please contact us at:

openaccess@tue.nl

providing details and we will investigate your claim.
}

Copyright and moral rights for the publications made accessible in the public portal are retained by the authors and/or other copyright owners and it is a condition of accessing publications that users recognise and abide by the legal requirements associated with these rights.

- Users may download and print one copy of any publication from the public portal for the purpose of private study or research.

- You may not further distribute the material or use it for any profit-making activity or commercial gain

If the publication is distributed under the terms of Article $25 \mathrm{fa}$ of the Dutch Copyright Act, indicated by the "Taverne" license above, please 


\title{
Production of high-quality electron bunches by dephasing and beam loading in channeled and unchanneled laser plasma accelerators ${ }^{\text {a) }}$
}

\author{
C. G. R. Geddes, ${ }^{\text {b) }}$ Cs. Tóth, J. van Tilborg, ${ }^{\text {c) }}$ E. Esarey, and C. B. Schroeder \\ Lawrence Berkeley National Laboratory, 1 Cyclotron Road, Berkeley, California 94720 \\ D. Bruhwiler, C. Nieter, and J. Cary ${ }^{\mathrm{d})}$ \\ Tech-X Corporation, 5621 Arapahoe Avenue, Suite A, Boulder, Colorado 80303 \\ W. P. Leemans ${ }^{\mathrm{e})}$ \\ Lawrence Berkeley National Laboratory, 1 Cyclotron Road, Berkeley, California 94720
}

(Received 19 November 2004; accepted 27 January 2005; published online 18 April 2005)

\begin{abstract}
High-quality electron beams, with a few $10^{9}$ electrons within a few percent of the same energy above $80 \mathrm{MeV}$, were produced in a laser wakefield accelerator by matching the acceleration length to the length over which electrons were accelerated and outran (dephased from) the wake. A plasma channel guided the drive laser over long distances, resulting in production of the high-energy, high-quality beams. Unchanneled experiments varying the length of the target plasma indicated that the high-quality bunches are produced near the dephasing length and demonstrated that channel guiding was more stable and efficient than relativistic self-guiding. Consistent with these data, particle-in-cell simulations indicate production of high-quality electron beams when trapping of an initial bunch of electrons suppresses further injection by loading the wake. The injected electron bunch is then compressed in energy by dephasing, when the front of the bunch begins to decelerate while the tail is still accelerated. () 2005 American Institute of Physics. [DOI: 10.1063/1.1882352]
\end{abstract}

\section{INTRODUCTION}

Laser driven plasma wakefield accelerators have demonstrated high accelerating gradients of up to several hundred $\mathrm{GV} / \mathrm{m},{ }^{1-7}$ making them attractive as next generation sources of particles and radiation. ${ }^{8,9}$ The ponderomotive or radiation pressure of an intense laser pulse is used to drive a density oscillation (wake) in a plasma, the longitudinal field of which accelerates particles. ${ }^{8}$ Historically, electron beams produced had $100 \%$ energy spread ${ }^{1-7}$ limiting applications. Recently, however, experiments have produced beams of narrow energy spread by extending the distance of propagation using a guiding channel. ${ }^{10}$ Similar beams have also been observed using a large laser spot size to increase the diffraction range $Z_{R}=\pi w_{0}^{2} / \lambda$ with $w_{0}$ being the spot size, and hence the propagation distance of the laser pulse through the gas jet. ${ }^{11,12}$ Understanding and optimizing the physics responsible for producing these narrow energy spread beams is important to allow development of laser accelerators for radiation sources, ${ }^{13-15}$ high-energy physics, ${ }^{16}$ and other applications requiring small energy spread and stable beams.

Due to the periodic structure of the accelerating field of the wake, electrons injected at different phases (longitudinal positions behind the laser pulse) experience different accelerating fields, leading to large energy spread. While phase controlled optical injection techniques ${ }^{17-21}$ have been pro-

\footnotetext{
${ }^{a)}$ Paper HI2 2, Bull. Am. Phys. Soc. 49, 176 (2004).

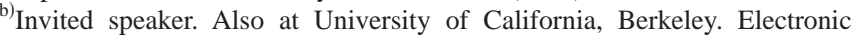
mail: cgrgeddes@lbl.gov

${ }^{c)}$ Also at Technische Universiteit Eindhoven, The Netherlands.

d) Also at University of Colorado, Boulder.

${ }^{\text {e)} U R L: ~ h t t p: / / l o a s i s . l b l . g o v ~}$
}

posed in order to reduce energy spread, these have not yet been fully demonstrated experimentally. Rather, current experiments, including those described above, use relatively high density plasmas, where the laser pulse is modulated to match the plasma period by the nonlinear response of the plasma (self-modulation ${ }^{22}$ ) and very high amplitude wakes are excited. When the wake reaches the so-called wave breaking amplitude, electrons are trapped from the bulk plasma and accelerated. ${ }^{1,5-7}$ The group velocity of the laser, and hence the phase velocity of the plasma wave is lower at high density, reducing the wave breaking threshold and allowing trapping of electrons at accessible laser intensities. Self-trapping such as this can inject particles over a wide phase range leading to the expectation that such accelerators would not produce beams of narrow energy spread, an expectation which was borne out by experiments until recently.

Here we present experiments, carried out at the L'OASIS facility of Lawrence Berkeley National Laboratory (LBNL), and simulations, with the code VORPAL, ${ }^{23}$ which show that high-quality electron bunches were formed in the selftrapped, self-modulated regime by a combination of beam loading and dephasing. We find that electron bunches of narrow energy spread and high energy are preferentially produced when the accelerator length matches the dephasing length $L_{d e p h}$ over which the electrons outrun the wake and slip into decelerating phase.

Section II describes channeled wakefield acceleration experiments, which produced the highest quality electron bunches observed by guiding the drive laser pulse using a plasma density channel to produce a long, controlled interaction length. Section III describes particle-in-cell simulations consistent with these experiments which indicate that 


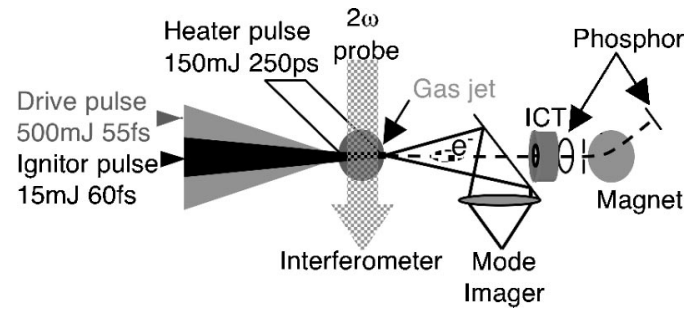

FIG. 1. Schematic of the channel guided wakefield accelerator, showing laser beams and principal diagnostics. A plasma is formed by an ignitor laser pulse in a pulsed hydrogen gas jet and heated by a heater pulse. A high intensity drive pulse is focused at the edge of the resulting plasma channel, in which it drives a plasma wave, accelerating electrons. Propagation of the laser is monitored with a side interferrometer and mode imager CCD. The electron beam is analyzed using an integrating current transformer (ICT), a phosphor screen, and a magnetic spectrometer.

beam loading, where the accelerated electrons alter the wake, allows the formation of the low energy spread bunch by turning off injection after the self-trapping of the initial group of electrons, effectively creating a self-consistent phased electron load. At the dephasing length, these particles are concentrated in energy because of their rotation in phase space, forming the narrow energy spread bunch. Section IV describes unchanneled experiments where plasma lengths less than, equal to, and greater than the dephasing length were used. Bunches with low energy spread were produced by matching the plasma length to the dephasing length, but these beams were less stable and produced less charge than channeled experiments at the same power. Section V summarizes the results and offers applications and opportunities for further development.

\section{CHANNELED WAKEFIELD ACCELERATION EXPERIMENTS}

A preformed plasma density channel was used to extend and control the focused propagation distance of the drive laser pulse in the plasma. The channel was created in a gas target by two laser pulses using a variant of the ignitor-heater method, ${ }^{24}$ with the multiarm L'OASIS Ti: sapphire laser. ${ }^{25,26}$ The laser operates at $800 \mathrm{~nm}$ with chirped pulse amplification, ${ }^{27}$ providing up to $10 \mathrm{TW}$ of laser power in a drive beam as well as secondary beams for channel formation and injection. For these experiments, a nearly uniform $2.4 \mathrm{~mm}$ long gas target at an atomic density near $4 \times 10^{19} \mathrm{~cm}^{-3}$ was provided by a supersonic hydrogen gas jet. A $60 \mathrm{fs}, 15 \mathrm{~mJ}$ ignitor pulse was focused at $f / 15$ near the downstream edge of the gas jet. The focal location and $f /$ number were chosen so that beam convergence roughly balanced ionization induced refraction, ${ }^{28,29}$ producing a cylindrical plasma of nearly uniform radius. This plasma was subsequently heated to tens of $\mathrm{eV}$ by inverse bremsstrahlung using $\sim 50 \mathrm{~mJ}$ from a $250 \mathrm{ps}, 150 \mathrm{~mJ}$ pulse cylindrically focused and incident on the jet from the side. This arrangement is shown in Fig. 1. Use of two channel formation pulses allowed both efficient ionization (requiring a high intensity pulse) and heating (optimized with a longer, low intensity pulse). ${ }^{24}$ The resulting hot plasma filament on axis expanded outward, driving a shock wave in the surrounding neutral gas. This shock resulted in a density depletion on axis and a nearly parabolic transverse density profile. ${ }^{30}$ Solution of the wave equation shows that such a profile can be matched to guide a Gaussian laser pulse without distortion in the low intensity limit where relativistic effects are not important. For a density rise of $\delta n=1 / \pi r_{e} r_{s}^{2}$, over a spot size $r_{s}$ and where $r_{e}$ is the classical electron radius, the change in phase velocity induced by rising density just counters diffraction, and the laser pulse remains collimated. Guiding at intensities up to $2 \times 10^{17} \mathrm{~W} / \mathrm{cm}^{2}$, which are too low to trap and accelerate electrons, has been demonstrated by previous experiments using this method. ${ }^{24,31-34}$

\section{A. Guiding of relativistically intense laser pulses in plasma channels}

Guiding at high intensity, as desired for wakefield acceleration, requires controlling both diffraction and relativistic modification of the plasma refractive index. ${ }^{35}$ Relativistic self-guiding occurs when the laser is intense enough that the quiver motion of the electrons causes their mass to increase, changing the refractive index. ${ }^{36-39}$ This effect, which occurs in regimes of interest to wakefield acceleration, provides some self-guiding for laser powers above the critical power $P_{c}$, but is unstable, eventually destroying the pulse and limiting propagation distance. ${ }^{35,40,41}$ In the present experiments, the drive pulse is of relativistic intensity, and so the channel profile was detuned from the low power matching condition, with a smaller rise in density to compensate for the presence of self-guiding.

The density profile of the channel has been matched to guide the drive pulse by choosing the plasma temperature (which determines shock velocity) using the heater beam and by adjusting the timing of the guide pulses. The drive pulse, which was collinear with the ignitor and focused near the entrance of the channel, had a $55 \mathrm{fs}$ pulsewidth full width at half maximum (FWHM), with powers of 0-9 TW and a focused spot size of $7-8.5 \mu \mathrm{m}$. To tune the channel, the plasma and its evolution were characterized interferometrically using a frequency doubled (405 $\mathrm{nm}$ wavelength) pulse incident from the side, with femtosecond temporal resolution. An imaging system using cylindrical optics provided spatial resolution of $17 \times 4 \mu \mathrm{m}^{2}$, to allow viewing of the long narrow plasma channels. The phase map from the interferometer was Abel inverted to recover the electron density assuming a cylindrically symmetric distribution. Symmetry is confirmed by the round mode structure of the guided laser pulse (below).

Drive pulses below the self-guiding threshold were well guided by channels with radial density profiles tuned to match the low power guiding condition described above. As power was increased above the self-guiding threshold, the output mode distorted in such matched channels, and the channel was adjusted to compensate for the presence of selfguiding by reducing the radial density gradient. Guiding of drive pulses up to $4 \mathrm{TW}\left(2 P_{c}\right)$ has been obtained without aberration of the laser mode and without self-trapping of electrons. ${ }^{42}$ This provides a long scale length structure for experiments on controlled injection which may stabilize and increase quality of beams from laser accelerators. ${ }^{17,18,21}$ 

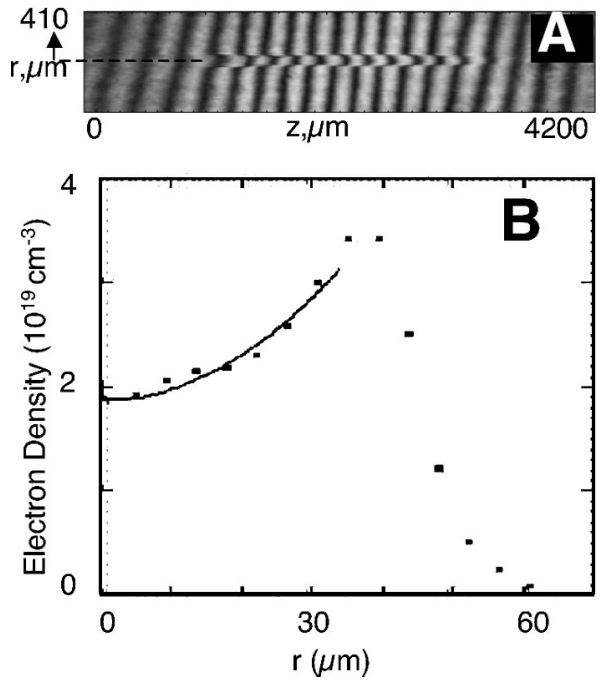

FIG. 2. Plasma channel formed by the ignitor and heater pulses at the time of arrival of the drive pulse. A frequency doubled interferrometer images the plasma transversely (a). The transverse density profile (b) was obtained from Abel inversion of the resulting phase map with cylindrical symmetry about the $z$ axis, showing data (black dots) and a parabolic fit (line).

Above 4 TW the beam is still well guided, but some distortion of the laser mode was always present (see below) due to the strong contribution of self-guiding.

Optimal guiding of the drive pulse at $9 \mathrm{TW}$, and optimal acceleration of self-trapped electron bunches, was observed in channels having an axial density of $1.9 \times 10^{19} \mathrm{~cm}^{-3} \pm 10 \%$ over the central $1.7 \mathrm{~mm}$ of the jet. The length of the wake drive laser pulse was thus longer than the linear plasma period, providing operation in the selfmodulated regime. ${ }^{22,38}$ This allowed the channels to extend laser propagation distance and enhance acceleration performance in self-modulated wakefield accelerators, where high densities allow self-trapping and acceleration of electrons. ${ }^{10,43}$ The profile of the channel, shown in Fig. 2, had a $\sim 40 \%$ reduced rise in density over a laser spot size compared to the matched profile for a low intensity pulse. ${ }^{24}$ This detuning was required to optimize guiding of the 9 TW pulse due to the contribution of self-guiding for this power.

The plasma channel guided the drive pulse, maintaining its intensity and nearly eliminating diffraction over $10 Z_{R}$, as shown in Fig. 3. After the propagation of the $9 \mathrm{TW}$ drive pulse, the side interferometer image [Fig. 3(a)] showed a plasma only slightly wider than the original channel diameter [Fig. 2(a)]. This indicates that the drive pulse was well confined within the channel since leakage of the drive pulse from the channel ionizes the surrounding neutral gas. The spatial mode of the laser pulse was characterized using a mode imaging camera, which could be translated to image the input or output of the channel. This mode image [Fig. $3(\mathrm{~b})]$ showed an intense spot of $24 \mu \mathrm{m}$ FWHM containing $10 \%$ of the input energy, confirming that the drive pulse was well guided. The spot is circular, confirming that the guiding channel is cylindrically symmetric. The reduction by a factor of 10 in energy from the input spot is due to a combination of leakage from the channel and depletion of the laser energy to excite the wakefield. Enlargement of the spot compared to
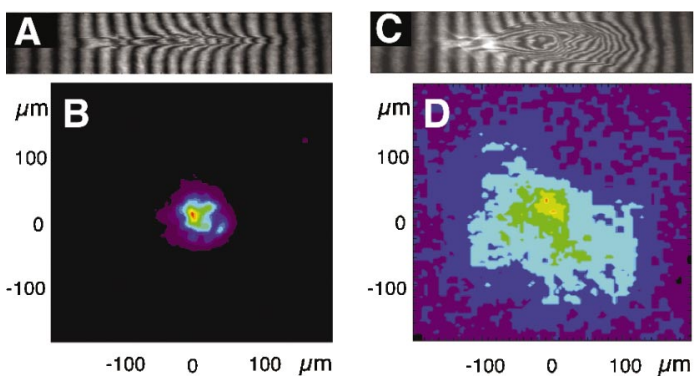

FIG. 3. (Color). Propagation of the laser through the gas jet was measured by the side looking interferrometer (a,c) and mode (laser spot) imager (b,d). In the channel guided accelerator, the plasma interferrogram after the passage of the drive beam (a) was similar to the guiding channel [see Fig. 2(a)], indicating that the drive laser pulse is confined to the channel. The laser mode at the channel exit is a well defined spot of $24 \mu \mathrm{m}$ FWHM after 2.4 $\mathrm{mm}$ of propagation (b). When the channel is off, the interferrogram (c) shows a plasma expanding rapidly along the propagation direction (left to right). The mode image (d) shows a diffuse transmitted spot. This indicates the effectiveness of the channel in maintaining the drive beam intensity and mode over many diffraction lengths.

the $8.5 \mu \mathrm{m}$ input and the leakage outside the guide appeared as the input intensity was increased beyond two times the relativistic self-focusing threshold. This is believed to be due to the inability of the channel to perfectly control the spot size in the presence of intense self-guiding. The enlargement due to this effect is small, however, compared to the diffraction displayed when the channel is off. In this case, the interferometer [Fig. 3(c)] showed a plasma which expands rapidly in the propagation direction, indicating that the laser is diffracting. The laser mode image [Fig. 3(d)] showed a diffuse transmitted spot with a diameter near $100 \mu \mathrm{m}$. Correlation of the mode imager and interferometer measurements showed that an intense guided mode was present only when the interferometer indicated that the laser was well confined to the channel. We next describe the effect of using a preformed plasma channel on the electron acceleration process.

\section{B. Electron acceleration in plasma channels}

Electrons trapped and accelerated by the plasma wave were momentum dispersed using a magnetic spectrometer with a bend angle of $55^{\circ}$ to obtain the electron energy spectrum. The electron beam was then detected by a phosphor screen imaged onto a charge coupled device (CCD) camera (see Fig. 1). The energy range covered in a single shot was $\pm 15 \%$ about a central energy selectable from 0 to $80 \mathrm{MeV}$, allowing structure in the energy distribution to be observed on a single shot basis. A full spectrum from 0 to $92 \mathrm{MeV}$ averaged over many shots was obtained by scanning the magnet current. The multishot spectrum allowed determination of the peak energy achieved and shot-to-shot statistics at a given energy. A slit was used at the input of the spectrometer to give $\pm 2 \%$ energy resolution. To detect electrons above $92 \mathrm{MeV}$, a separate phosphor observing bend angles of 0 to 5 was used. Because of the small bend angle and observation down to $0^{\circ}$, this diagnostic could observe electrons of arbitrarily high energy, but could not resolve the energy spread of the beam. In either configuration, the beam divergence was obtained in the undispersed direction. The magnetic 


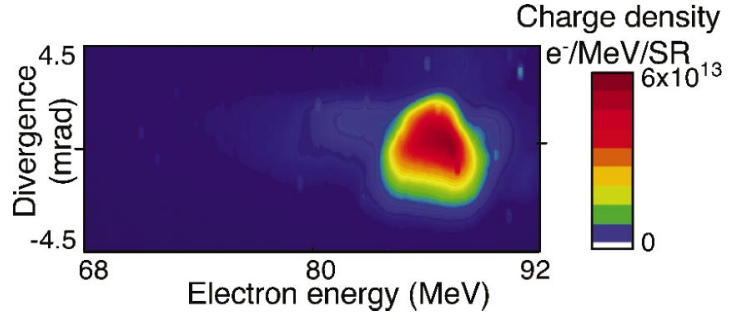

FIG. 4. (Color). Single shot electron beam spectrum of the channel guided accelerator, showing a strongly peaked distribution, in contrast to the exponential distribution of the unchanneled accelerator. A contour plot of electron beam intensity vs. energy and divergence shows a bunch containing $2 \times 10^{9}$ electrons in a narrow distribution at $86 \pm 1.8 \mathrm{MeV}$ and $3 \mathrm{mrad}$ divergence FWHM, with contrast $>10: 1$ above background.

spectrometer was calibrated against an integrating charge transformer, located before the slit and subtending $f / 8$, to obtain the charge in the electron beam. To measure the spatial shape of the electron beam and to provide a divergence measurement integrated over all energies, a beam phosphor screen (BPS) could be inserted prior to the magnet, showing the spatial profile of the energy integrated beam.

The magnetic spectrometer showed that the channel guided accelerator produced electron bunches with a large fraction of the charge in a well defined bunch at $86 \mathrm{MeV}$ with $3.6 \mathrm{MeV}$ energy spread FWHM and 3 mrad divergence FWHM (Fig. 4), in contrast to previous experiments which observed beams of continuous energy spread. The BPS confirmed that the channel guided accelerator [Fig. 5(a)] produces collimated beams, with divergence near $6 \mathrm{mrad}$ FWHM (integrated over all energies). The beam is nearly circular, so that the one-dimensional divergence measurement from the spectrometer is representative, and the divergence of the bunch at $86 \mathrm{MeV}$ (Fig. 4) was half that of the integrated beam observed on the BPS, consistent with previous experiments that have shown that higher energy electrons were more collimated. ${ }^{6}$ The energy spread of the peak, at $\pm 2 \%$, is essentially limited by the spectrometer resolution so that the beam may in fact have narrower energy spread. The peak at $86 \mathrm{MeV}$ shows a contrast ratio greater than 10:1 above a broad distribution of charge extending on either side. To determine the charge of this high-energy bunch, the spectrometer current was scanned to obtain a full spectrum from 1 to $90 \mathrm{MeV}$ averaged over 300 shots. The integral of this
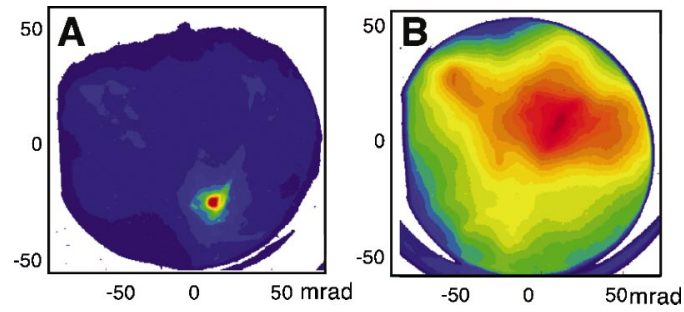

FIG. 5. (Color). Profile of the electron beam, showing the spatial distribution $72 \mathrm{~cm}$ after exiting the plasma. The profile was measured using a phosphor screen placed in the beam, imaged by a CCD camera. The channel guided accelerator (a) produces collimated beams with divergence near 6 mrad FWHM. The unchanneled accelerator produced diffuse electron beams with a much wider divergence of $50 \mathrm{mrad}$ FWHM (b). average spectrum was calibrated to the integrating charge transformer (ICT), accounting for the beam size and for the average overlap of the beam with the spectrometer slit as observed on the BPS. This calibration was verified by changing the slit size and observing that the amplitude of the spectrum changed by the expected ratio. The calibration of the magnetic spectrometer has also been verified by nuclear activation measurements. ${ }^{52}$ This provides a lower limit on the charge in the $86 \mathrm{MeV}$ beam of $2 \times 10^{9}$ electrons, assuming $100 \%$ transmission through the slit.

To obtain an estimate for the emittance it is first noted that space charge effects should be minimal for this relativistic beam. Assuming ballistic propagation from a source the size of the laser spot in the channel $(\sim 8.5 \mu \mathrm{m}$ at entrance, $\sim 24 \mu \mathrm{m}$ at end) is then a reasonable first approximation. The $3 \mathrm{mrad}$ divergence of the high-energy beam then indicates a geometric rms emittance $\epsilon_{x}$ of $0.005 \pi-0.01 \pi \mathrm{mm}$ mrad and a normalized emittance of $1 \pi-2 \pi \mathrm{mm}$-mrad rms, comparable to the best state of the art rf facilities. ${ }^{16}$ The above represents an upper limit, as it is likely the electron beam comes from a source smaller than the initial laser spot size (simulations, below, indicate a source size near $4 \mu \mathrm{m}$ ) indicating that a normalized emittance as small as $0.5 \pi \mathrm{mm} \mathrm{mrad}$ is possible.

The quality of optical guiding as well as the pointing, quality, and charge of the electron beams at high energy fluctuated from shot to shot, probably caused by laser pointing jitter that changes the overlap between the wake drive pulse and the channel formation pulses (and hence guide quality and incoupling) as well as laser power fluctuations. Beams with $3 \times 10^{9}$ electrons have been observed at similar energies ( $78 \pm 3 \mathrm{MeV}$ FWHM), and electrons were observed up to the limit of our $55^{\circ}$ high-resolution spectrometer $(92 \mathrm{MeV})$. Using the $5^{\circ}$ spectrometer, we observed bunches at energies of $150 \mathrm{MeV}$, but this diagnostic did not allow the fine resolution required to resolve energy spread. Structure in the energy spectrum has been seen for electrons with energy as low as $15 \mathrm{MeV}$. Below $15 \mathrm{MeV}$ there was an essentially continuous distribution, and total beam charge was $1.7 \times 10^{10}$ electrons as measured by the ICT, subtending $f / 8$. Using a bend magnet, the low-energy contribution can be separated, leaving a high-energy, high-quality beam with a few times $10^{9}$ electrons. Consistent control of guiding and electron injection ${ }^{17-21}$ in order to stabilize these beams are among the next challenges for laser accelerators.

\section{Correlations between accelerated electron yield and laser beam properties}

Well guided optical beams were highly correlated with the acceleration of electrons to high energies. Simultaneous observation of the mode image and the $5^{\circ}$ spectrometer over 300 shots allowed measurement of the correlation between guided mode intensity and charge of the electron beam at high energy. Figure 6 shows the charge at energy greater than $100 \mathrm{MeV}$ as a function of normalized guided mode intensity. Normalized guided mode intensity is defined as the peak intensity in the mode image on a given shot normalized to the peak observed on any shot in the run, and parametrizes 


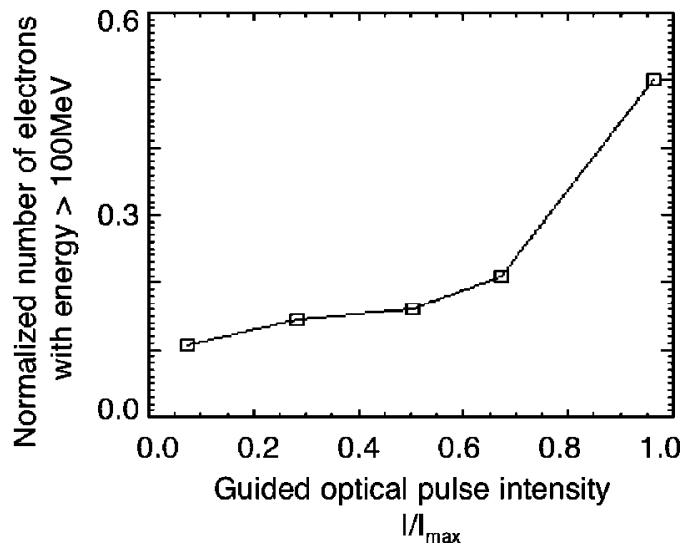

FIG. 6. Normalized electron charge above $100 \mathrm{MeV}$ vs guided mode intensity. Data are binned from 300 shots and normalized to the highest values observed during the run. High-energy beams are highly correlated with high-guided mode intensities, indicating that maintaining the drive laser intensity over the length of the plasma using the channel enhances accelerator performance.

the effectiveness of the guide in maintaining drive amplitude. Charge is measured by integrating the signal on the phosphor screen, normalized to the maximum charge observed. Data have been binned by guided mode intensity in order to display the dependency. There is a stronger than linear rise of charge with intensity, indicating that good mode transport was very important to high-energy electron production, or alternatively, that maintaining drive intensity over long distance is an essential criterion for obtaining high-energy electrons. This also is suggestive of the importance of matching the pump depletion distance to the dephasing distance to maximize the accelerator efficiency, though a detailed study of that issue is beyond the scope of the present paper.

We have also studied the correlation between the laser spectrum at the exit of the plasma channels and the generation of high-energy electron beams. To analyze the transmitted laser spectra, we divided each measured spectrum into an unshifted (within the laser bandwidth) region, and redshifted and blueshifted bands. The correlation of transmitted energy to high-energy electrons was positive, and the correlation was more than twice as strong for redshift as blueshift. Normalizing to spectrally integrated transmission, shots with high charge above $100 \mathrm{MeV}$ had 6\% more energy in the spectral lobe redshifted from the fundamental and 5\% less blueshifted energy. Hence, in contrast to previous reports, ${ }^{6}$ here we observe that the largest plasma waves (indicated by accelerated electrons) were most strongly correlated with redshifting of the laser light. This observation is consistent with fluid simulations and with energy conservation, which indicate that the laser must redshift as it deposits energy in the plasma wave.

To differentiate the effects of channeling from preionization, the ignitor pulse was fired 20 or 80 ps before the drive pulse, preionizing a plasma $80 \mu \mathrm{m}$ in diameter. The plasma did not expand significantly over $80 \mathrm{ps}$. Hence there was no shock wave and the transverse density profile was flat at $4 \times 10^{19} \mathrm{~cm}^{-3}$ and had no guiding properties. We observed no substantial difference between the drive pulse only (un-
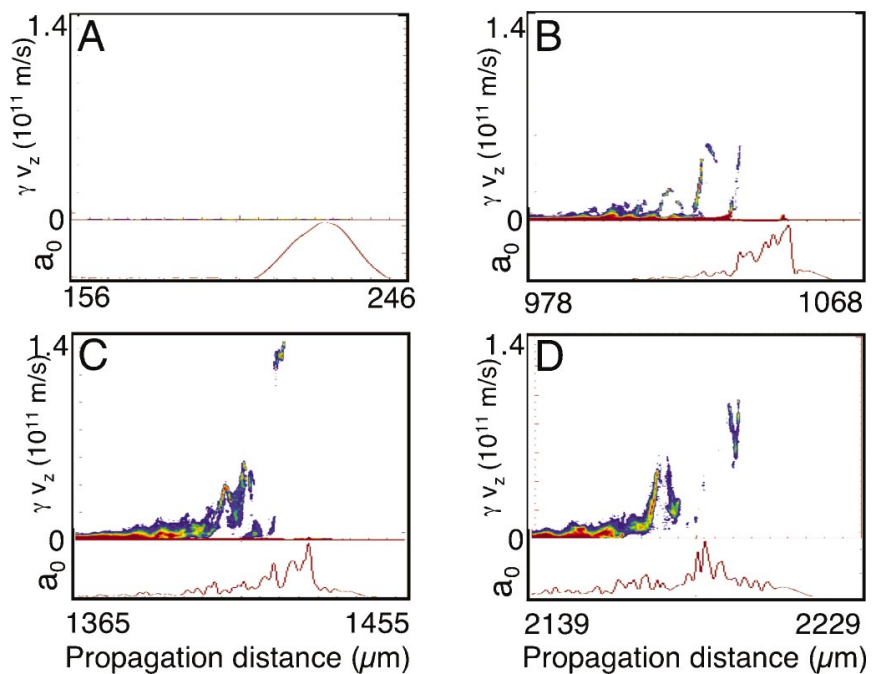

FIG. 7. (Color). Simulation momentum phase space (top of each panel) and laser envelope (bottom) as a function of propagation distance, $z[\mu \mathrm{m}]$. The laser enters the plasma (a), and is modulated by the plasma response, exciting a wake and trapping electrons (b). If trapping turns off after the initial bunch is loaded (see Fig. 8), these electrons are concentrated in energy at the dephasing length, forming a high-energy, low-energy spread bunch (c) which dissipates with further propagation (d).

channeled, below) and preionized cases in laser propagation, electron yield, or energy spectrum. This indicates that channeling and not preionization was responsible for differences in the electron beams described above, and is also consistent with the expectation that the plasma in the single beam experiment is ionized so early in the pulse that the bulk of the pulse (more than 99.9\%) sees an ionized target and is not significantly affected by ionization physics.

\section{SIMULATIONS OF BUNCH FORMATION}

Two-dimensional particle-in-cell (PIC) simulations using the code VORPAL (Ref. 23) were conducted to evaluate mechanisms of beam formation and to compare to the structure of the experimental electron spectra. Simulations were performed for the experimental densities and laser parameters, and analyzed as a function of propagation distance. The simulations used a moving window copropagating with the laser pulse, $90 \mu \mathrm{m}$ long in the longitudinal direction by $80 \mu \mathrm{m}$ transversely. The grid was $2700 \times 600$ cells with ten particles per cell. Plasma was loaded over the central $60 \mu \mathrm{m}$ of the box transversely. The laser is polarized out of the plane of the simulation.

The simulated laser envelope and particle phase space as a function of propagation distance are shown in Fig. 7, and the wake density is shown in Fig. 8, for the parameters of the channeled experiment. The laser initially enters the plasma with a pulse duration longer than the linear plasma period, and as a result does not excite a large wake or accelerate electrons [Fig. 7(a)]. As it propagates, the laser selfmodulates to the plasma period. This excites a wake ${ }^{9}$ of increased amplitude which can trap and accelerate electrons. Trapping and acceleration of a high-energy, high-quality beam was observed in the first plasma period of the wake (i.e., bucket) behind the laser, with electrons trapped when 


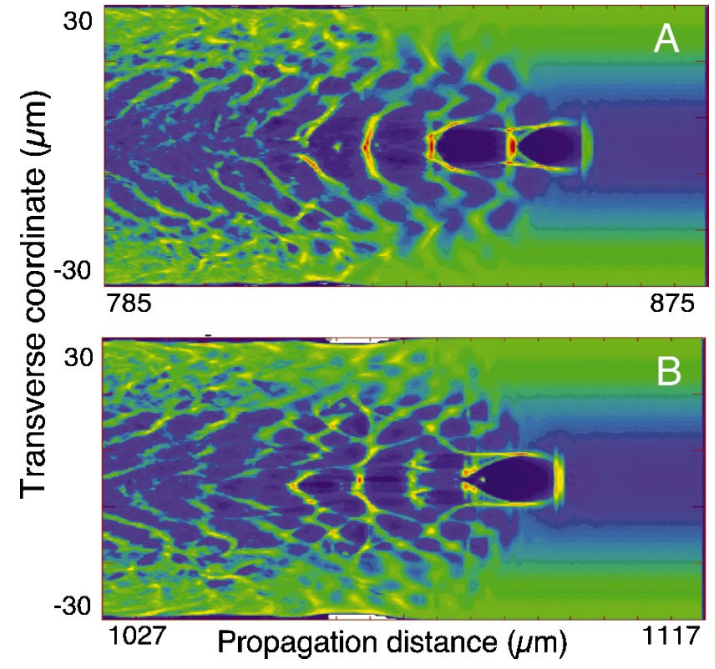

FIG. 8. (Color). Simulated electron density after laser propagation of 875 $\mu \mathrm{m}$ (a) and $1117 \mu \mathrm{m}$ (b), showing the wake and the effect of the trapped electrons on it. Just before trapping in the first bucket behind the laser (a), the wake structure is undisturbed and large in amplitude, allowing selftrapping of electrons. When a bunch is trapped, the wake is damped, suppressing further trapping and hence isolating the initial bunch in phase space (b). The bunch is visible in (b) as a small isolated green dot in the center of the first bucket, with density comparable to the plasma density. Buckets further behind the pulse trap and dephase earlier; accordingly, bunches are visible in trailing buckets before trapping in the first.

the laser has propagated $\approx 1 \mathrm{~mm}$ [Fig. 7(b)]. The field of the initial trapped electrons interfered with the wake driven by the laser, reducing its amplitude (beam loading). ${ }^{44}$ This effect can be seen in the reduction of wake amplitude from Figs. 8(a) (before trapping) to 8(b) (after trapping). Further trapping was suppressed by this loading. The initial electrons were then isolated in phase space and able to form a highquality bunch if suitably compressed in momentum space.

The electrons are compressed in momentum to form a high-quality beam at the dephasing length, ${ }^{9}$ the length at which the electrons outrun the wake and slip forward into a decelerating phase. As the leading edge of the bunch dephases and is decelerated, while the trailing edge is still accelerated, the particles are concentrated at the dephasing energy, which is also the maximum energy gain obtainable for the given parameters. In the simulation this results in the formation of a low-energy spread bunch after $1.5 \mathrm{~mm}$ propagation [Fig. 7(c)]. This optimum length is close to the 1.7 $\mathrm{mm}$ length of the high density plateau in the experimental plasma. Electron energy spread increases again with further propagation as the particles are decelerated, unless the plasma is terminated at this point. Peak electron energy remains nearly constant as the particles are mixed to fill all phases in the wake, but the number of electrons at high energy is reduced after $2 \mathrm{~mm}$ propagation [Fig. 7(d)]. The bunch dissipates more slowly than it forms since the laser pulse is depleting its energy into the wake as it propagates, resulting in reduced wake amplitude and slower deceleration, and monoenergetic features are present from $\sim 1.3$ to 1.9 $\mathrm{mm}$, with the energy of the peak varying from 80 to 220 $\mathrm{MeV}$. The asymmetry may be even greater in three dimensions, where filamentation and other instabilities may further disperse the laser, leading to observation of low-energy spread well beyond the dephasing length. Hence we expect that for plasmas shorter than the dephasing length lowenergy beams with continuous distributions would be observed, while at the dephasing length higher energies and structure (i.e., spikes of narrow energy spread) in the distribution would be present. After the dephasing limit, peak energy should not increase, and structure in the distribution and density of particles at high energy should be reduced. The peak energy observed in these simulations, $220 \mathrm{MeV}$, is close to the $150 \mathrm{MeV}$ observed in the experiments, and energy spread is likewise percent level in both cases. The simulations show less trapped charge than the experiments by a factor of 10, and comparison with other simulations suggests that this is due to a combination of resolution (trapped charge increased with longitudinal and particle resolution) and three-dimensional effects. Simulations with the laser polarized in the plane of the simulation have shown trapping of $10^{9}$ electrons (though with broader energy spread) under similar conditions, and this has also been observed in threedimensional simulations. ${ }^{45}$

Wake buckets behind the first one also trap particles, but these do not contribute to the highest energy beam. The difference may be due to transverse wave breaking and laser envelope effects. Due to the nonlinear variation of the plasma wave period with amplitude, the wake phase fronts curve, and curvature increases with increasing distance behind the laser pulse. This curvature reduces the threshold for trapping through transverse wave breaking for buckets far behind the pulse. ${ }^{46}$ The trapping threshold has also recently been shown to increase for buckets within the laser envelope (i.e., the first bucket). ${ }^{47}$ Consistent with this, simulations indicate that in buckets far behind the laser pulse the wave breaks and picks up particles early and at low wave amplitude. The peak energy obtainable from the wakefield rises with wave amplitude, ${ }^{9}$ so these particles dephase early and at low energy, and do not contribute to the highest energy beam. However, as each bucket dephases, its electrons are bunched in at the dephasing energy for that bucket. These energies range from 13 to $100 \mathrm{MeV}$, compatible with the observation of structure in the experimental energy spectrum at lower energies. Such structure may result when fluctuations in guide quality terminate the wake early, near the dephasing of a particular bucket, or due to fluctuation in laser and plasma parameters. Other simulations have also observed the formation of structure in the energy distribution over long propagation distances. ${ }^{48-50}$

\section{DEPHASING EXPERIMENTS}

The dependence of electron bunch energy and energy spread on dephasing was studied experimentally by changing the length and density of the plasma target. Use of various target conditions allowed evaluation of accelerator performance for accelerator lengths greater than, equal to, and less than the dephasing length. The laser was focused to an intensity of $1.1 \times 10^{19} \mathrm{~W} / \mathrm{cm}^{2}$ in a spot of $8.5 \mu \mathrm{m}$ FWHM in hydrogen gas jets. The pulse length was $55 \mathrm{fs,} \mathrm{a} \mathrm{few} \mathrm{times}$ the linear plasma period for the densities used, in the self- 
modulated regime. ${ }^{22,38}$ As the laser entered the gas, its front edge ionized a plasma. As it propagated, the pulse modulated into subpulses at the plasma period, trapping particles, and accelerating them as described above. ${ }^{1,7,35}$ Channeling was not used for these studies, so that they also provide a comparison between channeled and unchanneled operations. To allow evaluation of the electron beam statistics, no slit was used in the magnetic spectrometer, which ensured that most electron beams are captured by the spectrometer acceptance. Momentum resolution is reduced, but bunching in the energy spectrum is still visible.

Variation of gas jet density was accomplished by variation of the backing pressure, while length was varied by using shaped nozzles. The jets were supersonic to produce short edge gradients and a plateau region of nearly constant density whose length was determined by the nozzle size. We used a slit gas jet which provided a plateau of $600 \mu \mathrm{m}$ or $4 \mathrm{~mm}$ long depending on rotation with respect to the laser axis. For intermediate length, a cylindrical jet with a $1.8 \mathrm{~mm}$ plateau was used. Operating height of the laser above the jets was $0.5-1 \mathrm{~mm}$, selected to avoid burning the jet with the laser, and was greatest for the longer jets to allow for laser divergence. These selections gave us plasmas 0.6 or $1.8 \mathrm{~mm}$ long at $4 \times 10^{19} \mathrm{~cm}^{-3}$ and 0.6 or $4 \mathrm{~mm}$ long at $2 \times 10^{19} \mathrm{~cm}^{-3}$. Only the lower density was possible in the $4 \mathrm{~mm}$ long plasma, because the greater operating height required for the long plasma allowed the gas to expand further.

Using these variations of jet length and density, acceleration experiments were conducted with plasma length greater, equal to, or less than the dephasing length. Dephasing length was evaluated using simulations, since the experiments were in the self-modulated regime in order to allow electron trapping from the background plasma. In this regime, the analytic solution for the linear dephasing length, $L_{d}=\lambda_{p}^{3} / \lambda^{2}$ where $\lambda_{p}$ is the plasma wavelength, ${ }^{9,51}$ is not adequate because of the need to account for modulation and trapping. The simulations described above were repeated without guiding for plasma densities of $2 \times 10^{19}$ and 4 $\times 10^{19} \mathrm{~cm}^{-3}$. The results are qualitatively similar to those described above. Self-modulation and dephasing are faster at higher densities, so that the dephasing length decreases and the peak energies drop. Self-modulation also occurs more quickly without channeling, as the focusing effect of the channel suppresses radial modulation. At $2 \times 10^{19} \mathrm{~cm}^{-3}$, dephasing length is $1200 \mu \mathrm{m}$. At $4 \times 10^{19} \mathrm{~cm}^{-3}$, dephasing length drops further to $600 \mu \mathrm{m}$. Hence at $4 \times 10^{19} \mathrm{~cm}^{-3}$ lengths of $0.6 \mathrm{~mm} \approx L_{\text {deph }}$ and $1.8 \mathrm{~mm}>L_{\text {deph }}$ were available, allowing comparison of beams at and after dephasing. Using the second density of $2 \times 10^{19} \mathrm{~cm}^{-3}$, and lengths of $0.6 \mathrm{~mm}<L_{\text {deph }}$ and $4 \mathrm{~mm}>L_{\text {deph }}$ allowed additional comparison of predephasing and postdephasing beams. Data from the two densities together characterized the nature of the beam before, at, and after dephasing.

The electron energy spectrum of the accelerator operated at a plasma density of $4 \times 10^{19} \mathrm{~cm}^{-3}$ is shown in Fig. 9, for plasma lengths of $0.6 \mathrm{~mm}$ and $1.8 \mathrm{~mm}$. The $1.8 \mathrm{~mm}$ plasma corresponds to the same gas jet conditions as in the channeled experiment. In contrast to the guided accelerator, there is little structure in the electron energy distribution, and the

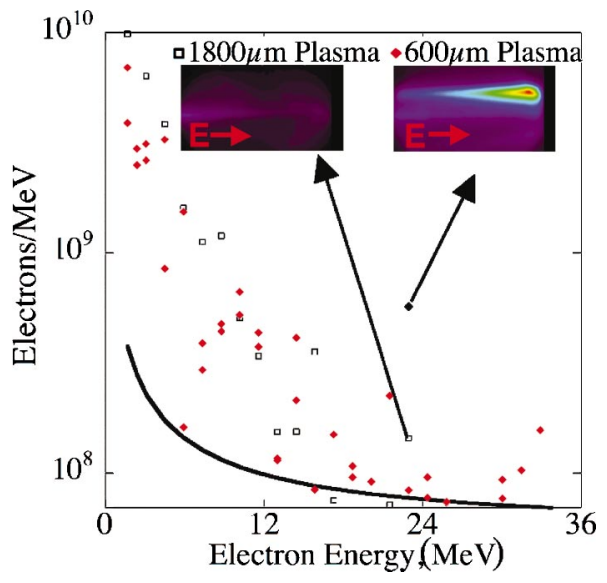

FIG. 9. (Color). Electron energy spectrum for the unchanneled accelerator operated at a density of $4 \times 10^{19} \mathrm{~cm}^{-3}$, obtained by scanning the magnet current over many shots. For a plasma length of $600 \mu \mathrm{m}$, near the dephasing length calculated in simulations, there is several times more charge at high energy than for a $1.8 \mathrm{~mm}$ long plasma, which is longer than the dephasing length. The insets show individual phosphor images, demonstrating that the spectrum is also more structured at the dephasing length, consistent with simulation. Fluctuation in the spectrum appears due to fluctuations in selfguiding and in laser parameters shot-to-shot.

peak energy is lower-below $40 \mathrm{MeV}$. The divergence of the beam is also much broader, near $50 \mathrm{mrad}$, as shown in Fig. 5(b). The peak energies observed in the averaged spectrum are similar at both plasma lengths, indicating that no acceleration occurred beyond $0.6 \mathrm{~mm}$. Individual phosphor images (Fig. 9, inset) show that there is substantially more structuring of the spectrum in the short plasma. Both features are consistent with a dephasing length near $600 \mu \mathrm{m}$ at this density, which is also close to the $600 \mu \mathrm{m}$ dephasing length seen in simulations. Beyond that length there should be little energy gain, and monoenergetic features should be washed out, as observed.

With a plasma density of $2 \times 10^{19} \mathrm{~cm}^{-3}$ the character of the energy spectra changed (Fig. 10), consistent with the increased dephasing length at the lower density. The averaged experimental energy spectrum for a plasma length of $0.6 \mathrm{~mm}$ shows low peak energies, while the individual phosphor images (inset) show no structure in the distribution. This is consistent with the electron beam being extracted well before dephasing. In contrast, the averaged spectrum at $4 \mathrm{~mm}$ plasma length shows that high energies are produced. Individual images show the appearance of monoenergetic structures in the spectrum at high energy. These features indicate the beam is extracted at or after dephasing. These data are also consistent with the dephasing length of $1200 \mu \mathrm{m}$ observed in simulations at this density.

The scaling of peak energy observed with density is roughly consistent with the theoretical scaling of maximum energy gain at dephasing. At dephasing, energy gain is proportional to the reciprocal of plasma density for constant $E_{\max } / E_{0}$, where $E_{\max }$ is the maximum accelerating field and $E_{0}$ is the wave breaking field. ${ }^{9}$ The peak energy observed at $2 \times 10^{19} \mathrm{~cm}^{-3}$ was above $90 \mathrm{MeV}$ at $4 \mathrm{~mm}$ propagation distance, compared to $40 \mathrm{MeV}$ at $4 \times 10^{19} \mathrm{~cm}^{-3}$. The same scaling of energies is observed in the simulations, which also 


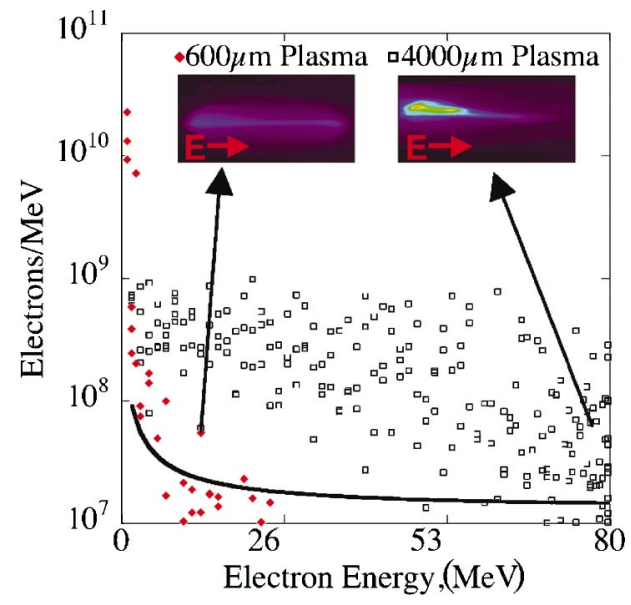

FIG. 10. (Color). Electron energy spectrum for the unchanneled accelerator operated at a density of $2 \times 10^{19} \mathrm{~cm}^{-3}$, obtained by scanning the magnet current over many shots. For a plasma length of $600 \mu \mathrm{m}$, before the dephasing length calculated in simulations, very low energies are produced compared to a $4 \mathrm{~mm}$ plasma, which is longer than the dephasing length. The insets show individual phosphor images, demonstrating that the spectrum is also unstructured and smooth before the dephasing point, consistent with the explanation that particles are bunched in energy at dephasing. Fluctuation in the spectrum appears due to fluctuations in self-guiding and in laser parameters shot-to-shot.

shows that $E_{\max } / E_{0}$ is the same for the two cases. The peak energies observed are roughly $60 \%$ of those obtained in simulations, possibly because the two-dimensional simulations do not model well instabilities such as filamentation that can disrupt the drive beam. The phosphor screen used in the spectrometer also has a high detection threshold of a few $10^{7}$, which limits detection.

Charge and energy of the beams fluctuated significantly in the unchanneled accelerator using the low density plasma. This may be due to instabilities in relativistic self-guiding, ${ }^{35}$ which was used to guide the laser pulse over the several millimeters (approximately ten diffraction ranges) required to achieve the dephasing condition in this case. Analysis of the laser mode transmitted through the plasma was performed using the mode imager camera. This showed that the transmitted laser profile was in fact highly unstable, as expected, often breaking into many beamlets. The instability of the beam may in fact be partly responsible for the production of high energy beams in the long plasma; even if the plasma is longer than the dephasing length, drive beam breakup and depletion may have stopped acceleration near dephasing on some shots. Hence, while the single beam experiment demonstrates the dephasing physics, in order to obtain stable beams at high energy use of a guiding channel will be important in order to obtain stable propagation.

Comparing the single beam experiments to experiments performed with the same laser and plasma density but using a guiding channel ${ }^{10}$ (see above), we find that the stability of the beam at high energy was greatly increased by the guiding channel, as expected from the analysis above. In the unchanneled case at a density of $2 \times 10^{19} \mathrm{~cm}^{-3}$, most shots produced no detectable charge at $80 \mathrm{MeV}$ (detection threshold $10^{7}$ electrons using the phosphor screen of the magnetic spectrometer), and fluctuation in charge was two orders of mag- nitude. In the channeled accelerator, by contrast, at the same density almost all shots showed substantial charge at high energy, and fluctuation was less than one order of magnitude. Charge at high energy was also at least two times higher. This is consistent with the mode image observations above, and indicates the advantage of channeled experiments. The plasma channel greatly improves the intensity and reproducibility of the output optical mode image, which measures how effectively the drive pulse focus (and hence plasma wave amplitude) was retained through the plasma. Remaining fluctuation in the channeled case appears to result primarily from pointing jitter of the laser spot into the channel, and it should therefore be possible to reduce this fluctuation further with stabilization.

Consistent with the explanation presented here, monoenergetic beams have also been observed in experiments at other laboratories using a large laser spot size to increase $Z_{R}$ and hence the propagation distance of the laser pulse. ${ }^{11,12}$ Like the unchanneled experiments described above, this method is less efficient than use of a plasma channel in terms of amount of charge accelerated and electron bunch energy per laser power because large spot size is required to obtain long the $Z_{R}$ required. This reduces laser intensity, and hence wake amplitude and trapping.

Quality of the beams is also influenced by the reliance on beam loading to turn off injection at the appropriate time. This introduces a sensitive dependence on laser power and energy as well as plasma density. In the PIC simulations, variations as little as $10 \%$ in laser energy were sufficient to change or broaden the electron distribution. In the experiments, laser power and energy typically fluctuated by $\approx 10 \%$, and we observed fluctuation between continuous and structured energy spectra shot-to-shot, consistent with this explanation. Improvements in laser stability as well as controlled electron injection ${ }^{17-21}$ offer a path to stabilized electron bunches of even higher quality.

\section{CONCLUSIONS}

In conclusion, experiments at the L'OASIS facility of LBNL with channeled and unchanneled self-modulated wakefield accelerators have demonstrated that it is possible to produce structured energy spectra with significant fractions of the total charge in bunches with few $\mathrm{MeV}$ energy spread. Simulations and experiments varying target density and length indicate that the high-quality beams are obtained by taking advantage of beam loading to produce a selfconsistent phased injection of the electrons and by matching the acceleration length to the length over which the electrons dephase from the wake to reduce energy spread. At high densities this can be done using a very short gas jet, but this leads to low peak energies and low efficiency. At lower density, longer acceleration distances and higher peak energies are obtained. The long acceleration distance at low densities requires maintaining the intensity of the drive laser over millimeter scales, and channeled experiments then demonstrate a clear advantage in beam charge, quality, and stability.

The beams from channel guided accelerators such as those described here open up a new class of experiments 
with laser accelerators. Improvements in laser stability as well as controlled electron injection will help to further stabilize and improve the properties of the electron bunches. Production of radioisotopes can immediately benefit from the higher average charge at high energy. ${ }^{52}$ Beams with high spectral density at high energy will enable more efficient use of these accelerators as radiation sources for physics, chemistry, and biology. ${ }^{13,15}$ The bright and intrinsically ultrafast nature of the beams, in particular, will enable probing of time resolved phenomena with unprecedented resolution. As issues of stability and reproducibility are addressed, these beams may be of suitable quality to serve as injectors for high-energy physics applications. Due to their good emittance, these beams also may offer focusability and luminosity competitive with state of the art $\mathrm{rf}$ accelerators. Such beams may be injected into and postaccelerated in subsequent laser accelerator stages to produce compact multistage electron linacs.

\section{ACKNOWLEDGMENTS}

This work was supported by the U.S. Department of Energy under Contract Nos. DE-AC03-76SF00098, DE-FG0395ER40926, DE-FG02-01ER41178, DE-FG02-03ER83857, NSF under Grant No. 0113907, and the D.O.E. Office of Advanced Scientific Computing Research under the Scientific Discovery through Advanced Computing program. This research used resources of the National Energy Research Scientific Computing Center (Grant No. DE-AC03-76SF00098). C.G.R.G. was also supported by the Hertz foundation. C.G.R.G. thanks his faculty advisor Jonathan Wurtele, at UC Berkeley. The authors appreciate contributions from Gerry Dugan, Jerome Faure, Gwenael Fubiani, Bob Nagler, Kei Nakamura, Ned Saleh, Brad Shadwick, Leon Archambault, Michael Dickinson, Scott Dimaggio, Don Syversrud, Joe Wallig, and Nathan Ybarrolaza.

${ }^{1}$ A. Modena, Z. Najmudin, A. E. Dangor, C. E. Clayton, K. A. Marsh, C. Joshi, V. Malka, C. B. Darrow, C. Danson, D. Neely et al., Nature (London) 377, 606 (1995).

${ }^{2}$ K. Nakajima, D. Fisher, T. Kawakubo, H. Nakanishi, A. Ogata, Y. Kato, Y. Kitagawa, R. Kodama, K. Mima, H. Shiraga et al., Phys. Rev. Lett. 74, 4428 (1995).

${ }^{3}$ D. Umstadter, S.-Y. Chen, A. Maksimchuk, G. Mourou, and R. Wagner, Science 273, 472 (1996).

${ }^{4}$ C. Moore, A. Ting, K. Krushelnick, E. Esarey, R. F. Hubbard, H. R. Burris, C. Manka, and P. Sprangle, Phys. Rev. Lett. 79, 3909 (1997).

${ }^{5}$ D. Gordon, K. C. Tzeng, C. E. Clayton, A. E. Dangor, V. Malka, K. A. Marsh, A. Modena, W. B. Mori, P. Muggli, Z. Najmudin et al., Phys. Rev. Lett. 80, 2133 (1998).

${ }^{6}$ V. Malka, S. Fritzler, E. Lefebvre, M. M. Aleonard, F. Burgy, J. P. Chambaret, J. F. Chemin, K. Krushelnick, G. Malka, S. P. D. Mangles et al., Science 298, 1596 (2002).

${ }^{7}$ W. P. Leemans, P. Catravas, E. Esarey, C. G. R. Geddes, C. Toth, R. Trines, C. B. Schroeder, B. A. Shadwick, J. van Tilborg, and J. Faure, Phys. Rev. Lett. 89, 4802 (2002).

${ }^{8}$ T. Tajima and J. M. Dawson, Phys. Rev. Lett. 43, 267 (1979).

${ }^{9}$ E. Esarey, P. Sprangle, J. Krall, and A. Ting, IEEE Trans. Plasma Sci. 24, 252 (1996)

${ }^{10}$ C. G. R. Geddes, C. Toth, J. van Tilborg, E. Esarey, C. B. Schroeder, D. Bruhwiler, C. Nieter, J. Cary, and W. P. Leemans, Nature (London) 431, 538 (2004).

${ }^{11}$ J. Faure, Y. Glinec, A. Pukhov, S. Kiselev, S. Gordienko, E. Lefebvre, J.-P. Rousseau, F. Burgy, and V. Malka, Nature (London) 431, 541 (2004).

${ }^{12}$ S. P. D. Mangles, C. D. Murphy, Z. Najmudin, A. G. R. Thomas, J. L.
Collier, A. E. Dangor, E. J. Divall, P. S. Foster, J. G. Gallacher, C. J. Hooker et al., Nature (London) 431, 535 (2004).

${ }^{13}$ P. Catravas, E. Esarey, and W. P. Leemans, Meas. Sci. Technol. 12, 1828 (2001).

${ }^{14}$ R. W. Schoenlein, W. P. Leemans, A. H. Chin, P. Volfbeyn, T. E. Glover, P. Balling, M. Zolotorev, K. J. Kim, S. Chattopadhyay, and C. V. Shank, Science 274, 236 (1996).

${ }^{15}$ W. P. Leemans, C. G. R. Geddes, J. Faure, C. Toth, J. van Tilborg, C. B. Schroeder, E. Esarey, G. Fubiani, D. Auerbach, B. Marcelis et al., Phys. Rev. Lett. 91, 4802 (2003).

${ }^{16}$ X. J. Wang, X. Qiu, and I. Ben-Zvi, Phys. Rev. E 54, R3121 (1996).

${ }^{17}$ D. Umstadter, J. K. Kim, and E. Dodd, Phys. Rev. Lett. 76, 2073 (1996).

${ }^{18}$ E. Esarey, R. F. Hubbard, W. P. Leemans, A. Ting, and P. Sprangle, Phys. Rev. Lett. 79, 2682 (1997).

${ }^{19}$ C. B. Schroeder, P. B. Lee, J. S. Wurtele, E. Esarey, and W. P. Leemans, Phys. Rev. E 59, 6037 (1999).

${ }^{20}$ G. Fubiani, E. Esarey, C. B. Schroeder, and W. P. Leemans, Phys. Rev. E 70, 016402 (2004).

${ }^{21}$ C. Moore, A. Ting, S. McNaught, J. Qiu, H. Burris, and P. Sprangle, Phys. Rev. Lett. 82, 1688 (1999).

${ }^{22}$ J. Krall, A. Ting, E. Esarey, and P. Sprangle, Phys. Rev. E 48, 2157 (1993).

${ }^{23}$ C. Nieter and J. Cary, J. Comput. Phys. 196, 448 (2004).

${ }^{24}$ P. Volfbeyn, E. Esarey, and W. P. Leemans, Phys. Plasmas 6, 2269 (1999).

${ }^{25}$ W. P. Leemans, P. Volfbeyn, K. Z. Guo, S. Chattopadhyay, C. B. Schroeder, B. A. Shadwick, P. B. Lee, J. S. Wurtele, and E. Esarey, Phys. Plasmas 5, 1615 (1998).

${ }^{26}$ C. Toth, J. van Tilborg, C. R. Geddes, G. Fubiani, C. B. Schroeder, E. Esarey, J. Faure, G. Dugan, and W. P. Leemans, in High Power Laser Ablation V, Taos NM (SPIE, Bellingham, WA, 2004).

${ }^{27}$ D. Strickland and G. Mourou, Opt. Commun. 56, 219 (1985).

${ }^{28}$ W. P. Leemans, C. E. Clayton, W. B. Mori, K. A. Marsh, P. K. Kaw, A. Dyson, C. Joshi, and J. M. Wallace, Phys. Rev. A 46, 1091 (1992).

${ }^{29}$ R. Rankin, C. E. Capjack, N. H. Burnett, and P. B. Corkum, Opt. Lett. 16, 835 (1991).

${ }^{30}$ H. M. Milchberg, T. R. Clark, C. G. Durfee III, and T. M. Antonsen, Phys. Plasmas 3, 2149 (1996).

${ }^{31}$ C. G. Durfee III and H. M. Milchberg, Phys. Rev. Lett. 71, 2409 (1993).

${ }^{32}$ K. Kim, I. Alexeev, J. Fan, E. Parra, and H. Milchberg, Advanced Accelerator Workshop, Mandalay bay 2002 (AIP, New York, 2002), p. 646.

${ }^{33}$ E. W. Gaul, S. P. Le Blanc, A. R. Rundquist, R. Zgadzaj, H. Langhoff, and M. C. Downer, Appl. Phys. Lett. 77, 4112 (2000).

${ }^{34}$ Y. Ehrlich, C. Cohen, A. Zigler, J. Krall, P. Sprangle, and E. Esarey, Phys. Rev. Lett. 77, 4186 (1996).

${ }^{35}$ E. Esarey, P. Sprangle, J. Krall, and A. Ting, IEEE J. Quantum Electron. 33, 1879 (1997).

${ }^{36}$ A. Litvak, Sov. Phys. JETP 30, 344 (1969).

${ }^{37}$ P. Sprangle, C. M. Tang, and E. Esarey, IEEE Trans. Plasma Sci. PS-15, 145 (1987).

${ }^{38}$ E. Esarey, J. Krall, and P. Sprangle, Phys. Rev. Lett. 72, 2887 (1994).

${ }^{39}$ W. B. Mori, IEEE J. Quantum Electron. 33, 1942 (1997).

${ }^{40}$ Z. Najmudin, K. Krushelnick, E. Clark, S. Mangles, B. Walton, A. Dangor, S. Fritzler, V. Malka, E. Lefebvre, D. Gordon et al., Phys. Plasmas 10, 2071 (2003).

${ }^{41}$ A. B. Borisov, A. V. Borovskiy, O. B. Shiryaev, V. V. Korobkin, A. M. Prokhorov, J. C. Solem, T. S. Luk, K. Boyer, and C. K. Rhodes, Phys. Rev. A 45, 5830 (1992).

${ }^{42}$ C. G. R. Geddes, C. Toth, J. van Tilborg, E. Esarey, C. B. Schroeder, D. Bruhwiler, C. Nieter, J. Cary, and W. P. Leemans, Phys. Rev. Lett. (submitted).

${ }^{43}$ R. F. Hubbard, D. Kaganovich, B. Hafizi, C. I. Moore, P. Sprangle, A. Ting, and A. Zigler, Phys. Rev. E 63, 036502 (2001).

${ }^{44}$ T. Katsouleas, S. Wilks, P. Chen, J. M. Dawson, and J. J. Su, Part. Accel. 22, 81 (1987).

${ }^{45} \mathrm{~F}$. Tsung and W. B. Mori (private communication).

${ }^{46}$ S. V. Bulanov, F. Pegoraro, A. M. Pukhov, and A. S. Sakharov, Phys. Rev. Lett. 78, 4205 (1997).

${ }^{47}$ C. B. Schroeder, E. Esarey, B. A. Shadwick, and W. P. Leemans, in Advanced Accelerator Concepts, edited by V. Yakimenko (AIP, New York, 2004), pp. 564-570.

${ }^{48}$ F. S. Tsung, R. Narang, W. B. Mori, C. Joshi, R. A. Fonseca, and L. O. Silva, Phys. Rev. Lett. 93, 185002 (2004). 
${ }^{49}$ A. J. W. Reitsma, W. P. Leemans, E. Esarey, C. B. Schroeder, L. P. J. Kamp, and T. J. Schep, Phys. Rev. ST Accel. Beams 5, 051301 (2002).

${ }^{50}$ A. Pukhov and J. Meyer-ter-Vehn, Appl. Phys. B: Lasers Opt. 74, 355 (2002).
${ }^{51}$ E. Esarey and M. Pilloff, Phys. Plasmas 2, 1432 (1995).

${ }^{52}$ W. P. Leemans, D. Rodgers, P. E. Catravas, C. G. R. Geddes, G. Fubiani, E. Esarey, B. A. Shadwick, R. Donahue, and A. Smith, Phys. Plasmas 8, 2510 (2001). 